\title{
SCUBA Observations of High Redshift Radio Galaxies
}

Michiel Reuland, Huub Rottgering, Wil van Breugel

This article was submitted to Radio Galaxies - Past, Present and Future Conference, Leiden, The Netherlands, November 11-15, 2002

\section{March 11, 2003}

U.S. Department of Energy

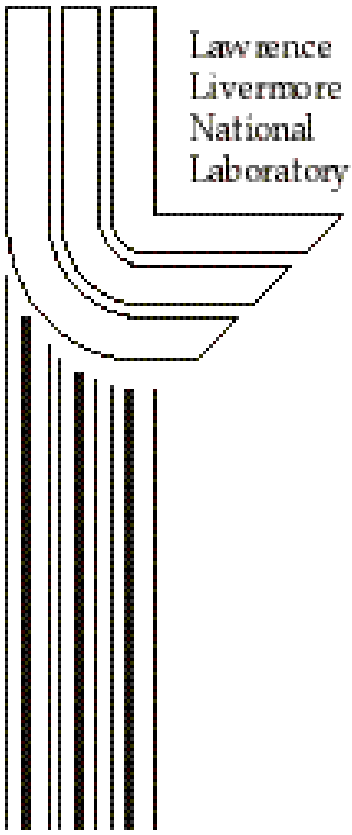


This document was prepared as an account of work sponsored by an agency of the United States Government. Neither the United States Government nor the University of California nor any of their employees, makes any warranty, express or implied, or assumes any legal liability or responsibility for the accuracy, completeness, or usefulness of any information, apparatus, product, or process disclosed, or represents that its use would not infringe privately owned rights. Reference herein to any specific commercial product, process, or service by trade name, trademark, manufacturer, or otherwise, does not necessarily constitute or imply its endorsement, recommendation, or favoring by the United States Government or the University of California. The views and opinions of authors expressed herein do not necessarily state or reflect those of the United States Government or the University of California, and shall not be used for advertising or product endorsement purposes. 


\title{
SCUBA Observations of High Redshift Radio Galaxies
}

\author{
Michiel Reuland ${ }^{\mathrm{a}, \mathrm{b}, \mathrm{c}}$ Huub Röttgering ${ }^{\mathrm{b}}$ Wil van Breugel ${ }^{\mathrm{a}}$ \\ ${ }^{\mathrm{a}}$ IGPP/LLNL, L-413, P.O. Box 808, Livermore, CA 94551, U.S.A. \\ ${ }^{\mathrm{b}}$ Leiden Observatory, P.O. Box 9513, 2300 RA Leiden, The Netherlands \\ ${ }^{\mathrm{c}}$ Department of Physics, UC Davis, 1 Shields Avenue, Davis, CA 95616, U.S.A.
}

\begin{abstract}
High redshift radio galaxies (HzRGs) are key targets for studies of the formation and evolution of massive galaxies. The role of dust in these processes is uncertain. We have therefore observed the dust continuum emission from a sample of $z>3$ radio galaxies with the SCUBA bolometer array. We confirm and strengthen the result found by Archibald et al. (1), that HzRGs are massive starforming systems and that submillimeter detection rate appears to be primarily a strong function of redshift. We also observed HzRG-candidates that have sofar eluded spectroscopic redshift determination. Four of these have been detected, and provide evidence that they may be extremely obscured radio galaxies, possibly in an early stage of their evolution.
\end{abstract}

Key words: galaxy formation, radio galaxies, dust, submillimeter emission

\section{Introduction}

The discovery of a tight relation between the masses of the stellar bulges of galaxies and the masses of their central black holes (2) has reemphasized the importance of high redshift active galactic nuclei (AGNs) as probes of galaxy formation. They are therefore the target of many studies of starformation in their host galaxies. Since a large proportion of the optical and UV emission from a starburst is absorbed and reprocessed by dust to restframe far-IR wavelengths, a good way to investigate the roles of dust and star formation in these distant galaxies is to measure their (sub-)millimeter continuum emission. Another advantage of observing at those wavelengths is that dimming due to cosmological distance is compensated for as the peak in the dust emission is redshifted into the observed passband (e.g., 3) 
The formation of massive elliptical galaxies may be best studied by tracing the evolution of powerful radio galaxies. They require the presence of an activelyaccreting super massive black hole $\left(\sim 10^{9} \mathrm{M}_{\odot} ; 4\right)$, are very extended and luminous at many wavelenghts allowing detailed studies, and their selection at radio wavelengths is not affected by the presence of dust.

Archibald et al. (1) have conducted a submillimeter survey of 47 radio galaxies over an interval of $0.7<z<4.4$. They found a substantial increase in $850 \mu \mathrm{m}$ detection rate with redshift and that the average $850 \mu \mathrm{m}$ luminosity $L_{850}$ rises at a rate $(1+z)^{3-4}$ out to $z \simeq 4$. While it is true that a buried AGN could be responsible for heating the dust, there are strong indications that starbursts are the dominating mechanism: (i) there is no strong correlation between radio power and submillimeter luminosity $(1 ; 5)$, (ii) for all hyperluminous infrared galaxies the starburst outshines the AGN at rest-frame wavelengths longwards of $50 \mu \mathrm{m}(6 ; 7)$, and (iii) the extent (up to $30 \mathrm{kpc}$ ) of the CO and dust-emission found in 3 HzRGs $(8 ; 9)$ is not readily explained by a central process.

Until recently only few HzRGs at $z>3$ were known, hampering statistical studies. However, using radio and near-IR selection we have significantly increased the sample of $z>3$ HzRGs at Keck (e.g., 10). Here we present submillimeter observations of these. Additionally, we have observed an intriguing class of HzRG-candidates for which no spectroscopic redshift could be determined. One of these candidates had been studied before in detail (WN J0305+3525; 11) and was found to be very submillimeter luminous.

\section{Sample Selection and Observations}

We have selected all known HzRGs with redshifts $z>3$ without prior SCUBA observations. We aimed to observe a significant sample of HzRGs to complement the observations of Archibald et al. (1) and obtain better statistical info at the highest redshifts. The sample is the result of an ongoing effort by our group and others (e.g., 10) to find distant radio galaxies based on Ultra Steep Radio Spectrum (USS) and near-IR identification selection criteria. Also 10 HzRG-candidates have been observed that did not show optical emission lines in deep spectroscopic observations at Keck.

The observations have been carried out over 4 semesters in 4 years (August 1999 - January 2002) with SCUBA (12) at the James Clerk Maxwell Telescope. We observed at $450 \mu \mathrm{m}$ and $850 \mu \mathrm{m}$ wavelengths in photometry mode. The optical depth $\tau_{850}$ varied between 0.14 and 0.38 with an average value of 0.26 . The data were reduced following standard procedures outlined in the SCUBA Photometry Cookbook. 


\section{Results and Discussion}

\subsection{HzRGs with $z>3$}

In the course of our program we observed $23 \mathrm{HzRGs,} \mathrm{of} \mathrm{which} 12$ were detected with a signal-to-noise ratio larger than 3 . Adding these data to the sample of Archibald et al. (1) increases the number of $3 \sigma$-detections at $z>3$ from 5 to 16. There was no attempt to correct for a contribution to the submillimeter continuum from the non-thermal radio emission, since most sources were selected using an USS criterion. Moreover, extrapolation from the radio regime to submillimeter wavelengths is very uncertain at best, and is likely to result in an overestimate of the non-thermal flux density due to steepening of the radio spectrum. For this reason (and following 14) we have chosen to use the "un-corrected" values from Archibald et al. (1). Figure 1 shows two histograms representing the number of HzRGs that were observed at each redshift and the number of HzRGs that were actually detected given a $2 \sigma$ or $3 \sigma$ detection criterion. Regardless of the actual detection limit used, a marked increase in the fractional detection rate from $\sim 15 \%$ at $z<2.5$ to $\sim 50 \%$ at $z>2.5$ is apparent, confirming the result found by Archibald et al. (1).

We have correlated the inferred submillimeter luminosities with radio power to search for signs of significant heating by the AGN. No strong correlation is apparent (Fig. 2). Recently, it was found that the size of the radio source and $L_{850}$ are correlated for a sample of radio-loud QSOs at $z=1.5$, with smaller (i.e. younger; 13) sources being more luminous (14). This effect was attributed to a possible relation between the jet-triggering event and a shortlived starburst or quasar-heated dust in QSOs. The effect was not found for a matched sample of radio galaxies at $z \sim 1.5$. We have checked whether there is a correlation between $L_{850}$ and radio source size (age) in our larger sample. Figure 2 shows that they are not strongly correlated. However, size of the radio source depends not only on age but also on jet-power and enviroment which is hard to correct for in our heterogeneous sample. Therefore we cannot exclude that $L_{850}$ and age of the radio source are correlated.

The (sub-)millimeter emission of starbursts should be largely indepent of viewing angle. Unification schemes (e.g., 15) therefore suggest that we can combine observations of HzRGs with those of QSOs, allowing us to study the evolution of starformation in their host galaxies over a larger redshift range. Figure 3 shows the average observed (sub-)millimeter fluxdensity as a function of redshift for mostly radio-quiet QSO samples $(16 ; 17 ; 18 ; 19)$ and HzRGs. Reassuringly, the trends mimic each other and they indicate that the average observed flux density rises to redshifts of $z \sim 4$ after which it declines. 

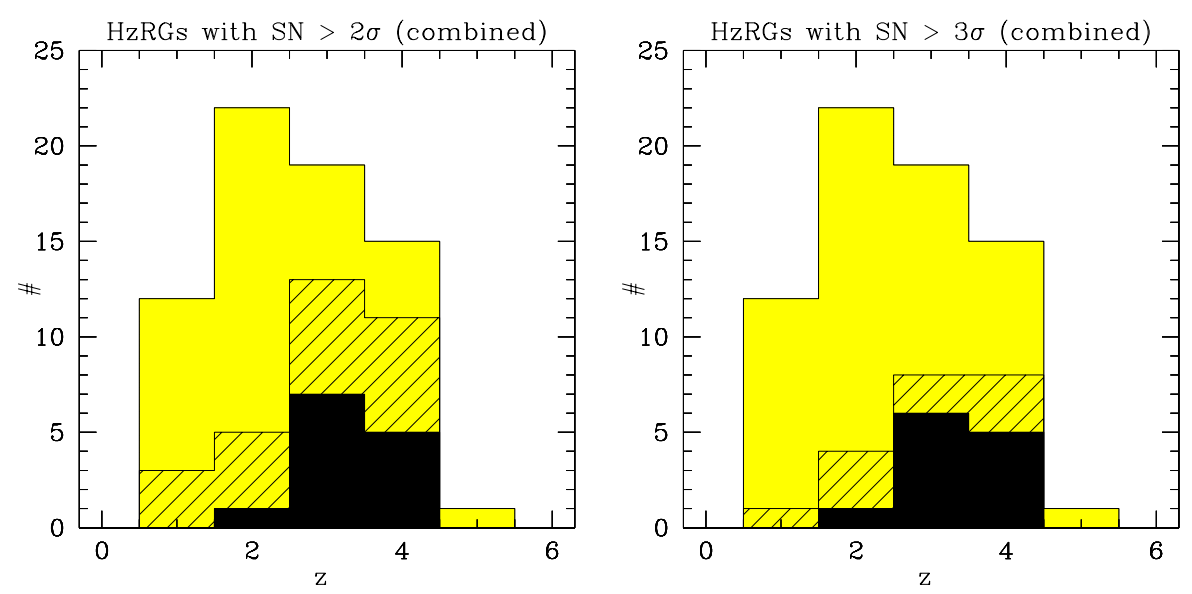

Fig. 1. Histograms of $850 \mu \mathrm{m}$ SCUBA detections (dashed) with $\mathrm{S} / \mathrm{N}>2$ (left) and $\mathrm{S} / \mathrm{N}>3$ (right) versus the total number of observed radio galaxies (grey) as a function of redshift. The data are from $(1 ; 5)$, with the detections from our program shown in black. At $z>2.5 \sim 50 \%-67 \%$ of the galaxies are detected, as opposed to $\sim 15 \%$ at $z<2.5$.
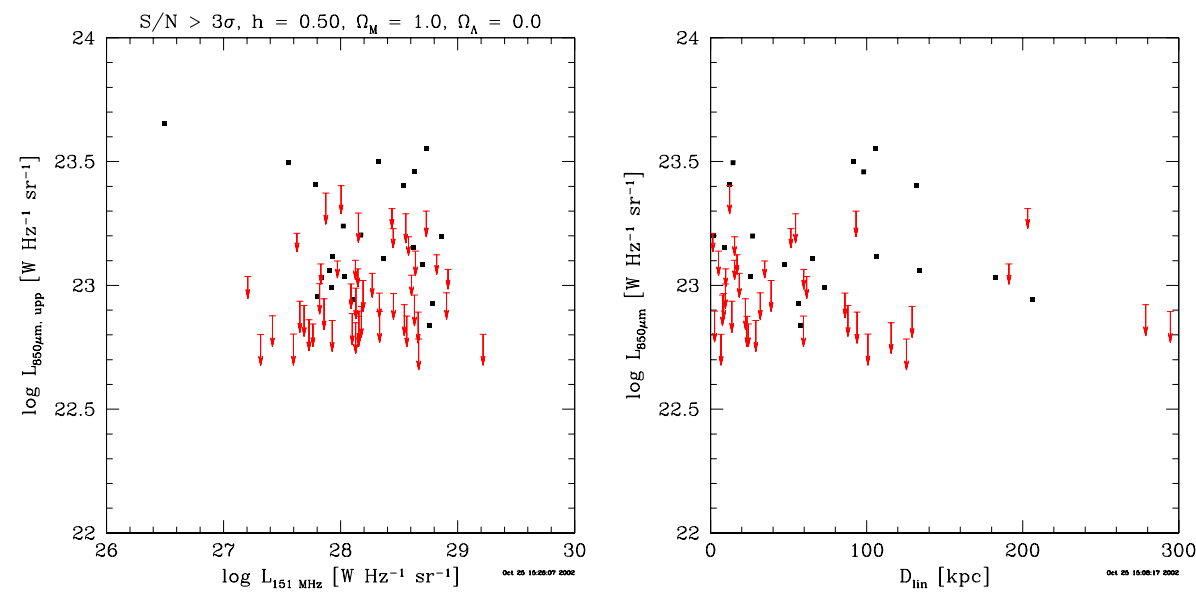

Fig. 2. The left plot shows $L_{850}$ versus radio power. The right plot shows $L_{850}$ versus linear size. No obvious correlations are present.

\subsection{Obscured "no-z" HzRGs}

During our search for HzRGs among USS sources, we found that $33 \%$ of the selected canditates were at redshifts $z>3(10)$. However we also found that $\sim 24 \%$, mostly with a compact radio morphology, do not show clear signs of emission lines and that a significant fraction $(\sim 10 \%$ of the entire sample) does not even show continuum emission. Possible explanations for this may be that (i) these are galaxies in the "redshift desert" $(1.5<z<2.3)$ where only weak emission lines fall in the optical window, (ii) they are pulsars, (iii) they are extremely obscured AGN (iv) or at such high redshift $(z>7)$ that Ly $\alpha$ is shifted out of the optical passband. 


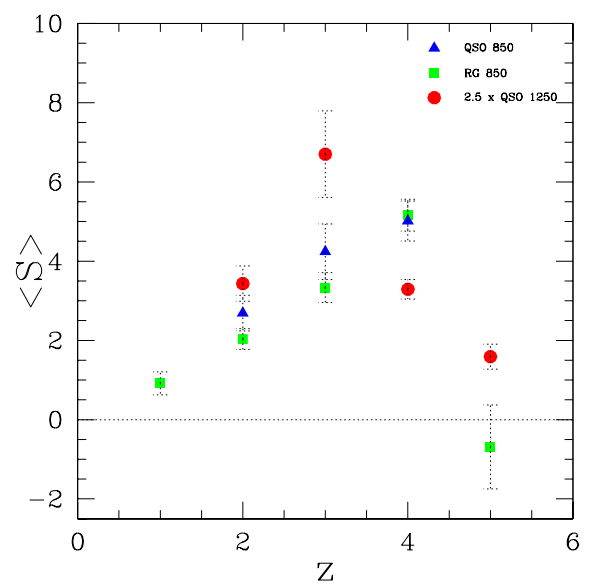

Fig. 3. The average measured (sub-)millimeter fluxdensity binned in redshift has been plotted for QSOs and radio galaxies. The squares represent observations of radio galaxies at $850 \mu \mathrm{m}$, the triangles QSOs at $850 \mu \mathrm{m}$, while the circles represent observations of QSOs at $1250 \mu \mathrm{m}$ multiplied by a factor of 2.5 for easier comparison.

Figure 4 shows one of these objects, WN J0305+3525, which was studied in detail (11). It is a strong (sub-)millimeter detection with $S_{850}=12.5 \pm 1.5 \mathrm{mJy}$ and $S_{1250}=4.2 \pm 0.6 \mathrm{mJy}$, and appears associated with a number of faint $K \sim 21 \mathrm{mag}$ and $J \geq 23 \mathrm{mag}$ objects. The radio morphology is compact $(\theta<$ $\left.1.9^{\prime \prime}\right)$, as is typical of the other candidates. Based on circumstantial evidence like the near-IR magnitudes and morphology, the (sub-)millimeter ratios and compact radio source morphology it was argued that WN J0305+3525 is a highly obscured HzRG possibly at a redshift $z=3 \pm 1$ in a young stage of its evolution (11). We have detected 3 more of these HzRG-candidates with flux densities of $S_{850} \approx 5 \pm 1$ mJy resulting in a detection rate of 4 out of 10 , very similar to what is found for the confirmed HzRGs. This further supports the idea that they may be young, extremely obscured HzRGs.

\section{Summary}

- Submillimeter detection rate appears to be primarily a function of redshift. If this is interpreted as being due to a change in the intrinsic far-IR luminosity, it would be consistent with a scenario in which the bulk of the stellar population of radio galaxies forms rapidly around redshifts of $z=3-5$ after which they are more passively evolving.

- A significant fraction (10\%) of USS sources may be very obscured radio galaxies. The obscuration may be related to their young evolutionary stage.

- In contrast to the submillimeter sources, HzRGs have accurately determined redshifts and host identifications. Even with the advent of SIRTF this is not likely to change. Therefore, HzRGs are a key population for studies of galaxy formation in the early universe, allowing detailed follow-up mminterferometry observations to study their dust and gas content. 

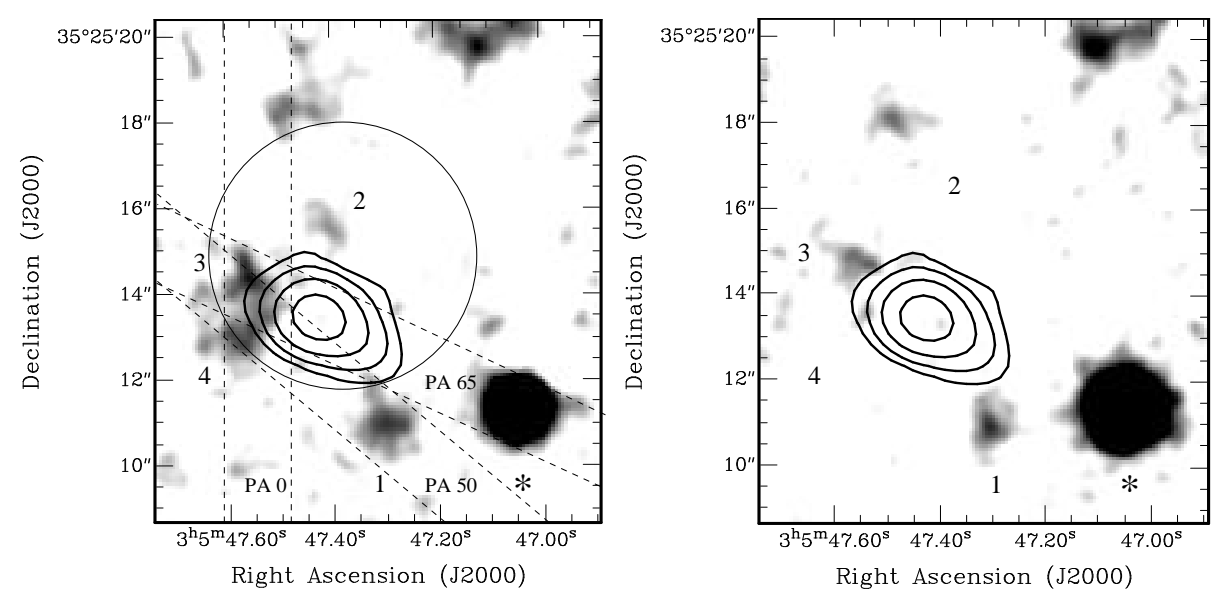

Fig. 4. Keck/NIRC $K$-band (left) and $J$-band (right) images with $4.85 \mathrm{GHz}$ VLA radio contours overlaid of the HzRG candidate WN J0305+3525. Note the multiple components; objects 2 and 4 are not detected in the $58 \mathrm{~min} J$-band image. The bright object to the SW is a spectroscopically confirmed star. The circle with $3^{\prime \prime}$ radius represents the nominal $3 \sigma$ astrometric uncertainty for the centroid of the $850 \mu \mathrm{m}$ emission and dashed lines indicate the LRIS slit positions.

The work of M.R. and W.v.B. was performed under the auspices of the U.S. Department of Energy, National Nuclear Security Administration by the University of California, Lawrence Livermore National Laboratory under contract No. W-7405-Eng-48.

\section{References}

[1] Archibald, E.N. et al., 2001, MNRAS, 323, 417

[2] Magorrian, J. et al., 1998, AJ, 115, 2285

[3] Blain, A. W. \& Longair, M. S. 1993, MNRAS, 264, 509

[4] Lacy, M. et al., 2001, ApJ, 551, L17

[5] Reuland, M. et al., in preparation

[6] Rowan-Robinson, M., 2000, MNRAS, 316, 885

[7] Farrah, D. et al., 2002, MNRAS, 335,1163

[8] Papadopoulos, P.P. et al., 2000, ApJ, 528, 626

[9] De Breuck, C. et al., 2002b, submitted.

[10] De Breuck, C., et al., 2001, AJ, 121, 1241

[11] Reuland, M. et al., 2003, ApJ, 528, 71

[12] Holland, W. et al., 1998, Proc. SPIE, 3357, 305

[13] Blundell, K., Rawlings, S. \& Willott, C. 1999, AJ, 117, 677

[14] Willott, C. J. et al.2002, MNRAS, 331, 435

[15] Barthel, P.D., 1989, ApJ, 336, 606

[16] Carilli et al., 2001, ApJ, 555, 625

[17] Omont et al., 2002, A\&A, accepted, astro-ph/0211655

[18] Isaak et al., 2002, MNRAS, 329, 149

[19] Priddey et al., 2002, MNRAS, in press, astro-ph/0211646 